Errata to the paper

\title{
ON THE PLANE SECTIONS METHOD FOR FUNCTIONS OF TWO VARIABLES
}

\author{
By T. L. McCOY
}

Quarterly of Applied Mathematics XXX, 481-490

The last paragraph of the proof of Lemma 4.1 is not correct. We provide here a corrected proof of the assertion that if $\rho\left(\lambda_{1}\right)=\infty$ and the $p_{n}$ are free of zeros in some neighborhood of $\lambda_{1}$, then $\rho(\lambda)=\infty$ in a full neighborhood of $\lambda_{1}$.

By hypothesis,

$$
\lim _{n \rightarrow \infty} \frac{1}{n} \log \left|p_{n}\left(\lambda_{1}\right)\right|=-\infty,
$$

an ordinary limit. Because the functions $(1 / n) \log \left|p_{n}(\lambda)\right|$ are harmonic and bounded above in a disc about $\lambda_{1}$, we can subtract off an appropriate constant and use Harnack's inequality ([1], p. 235) to infer the result. Indeed, it then follows from the subharmonic property of these functions that $\rho \equiv \infty$ in the entire plane.

We may as well mention that in the definition of $r(\lambda)$ in this paper, g.l.b. should be replaced by l.u.b.

\section{REFERENCES}

[1] Lars V. Ahlfors, Complex analysis, Second Edition, McGraw-Hill, 1966 